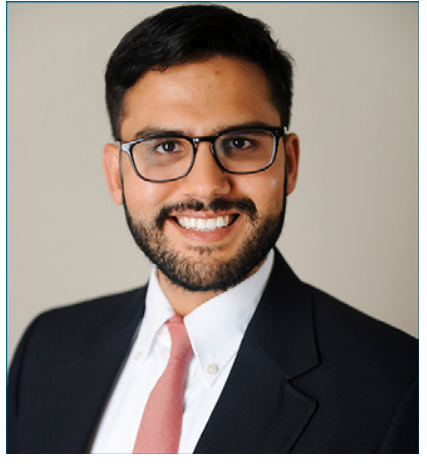

ALI RAZA KHAKI, MD

Ali Raza Khaki, MD, is a third-year Hematology/Oncology Fellow at the University of Washington. He completed medical school at University of California, San Diego, and residency at University of California, San Francisco. He is interested in health services and health outcomes research and clinical trial design.

Dr. Khaki has disclosed that he is supported by the $\mathrm{NCl}$ under award number T32CA009515.

\section{Clinical Risk During the Evaluation of Genomic Risk for Hormone-Sensitive Breast Cancer: Ignoring Valuable Data}

\author{
Ali Raza Khaki, MD; V.K. Gadi, MD, PhD; and Vinay Prasad, MD, MPH
}

$\mathbf{M}$ ultiple predictive assays have been implemented into clinical practice to help determe which women with early-stage hormone-sensitive breast cancer may benefit from adjuvant chemotherapy and which may not. These assays were originally developed as prognostic tools, and more recently have been used to guide deescalation of therapy. Before these assays were available, clinical tools such as Adjuvant! Online ${ }^{1}$ and PREDICT Plus ${ }^{2}$ were used for this purpose. These predictive tools relied on clinicopathologic markers from the patient and the primary tumor, including patient age, tumor size and grade, and number of involved lymph nodes. Conversely, novel assays use molecular technology-reverse transcription PCR (RT-PCR) or microarray gene expression profiling of a restricted set of targets-for prediction. Although many studies have asked whether molecular assays have predictive value, unfortunately few have studied whether assay-based predictive tools show benefit beyond that of clinicopathologic markers alone. This is a key question.

The 2 most prominent assays commercially available are Oncotype DX and MammaPrint, and both have phase III trial data supporting their use. Oncotype DX is a 21-gene assay using RT-PCR on surgically resected breast cancer tissue to provide a recurrence score (RS) predicting a women's risk of breast cancer recurrence. MammaPrint uses microarray gene expression profiling with a 70-gene breast cancer signature on surgically resected breast cancer tissue to classify tumors with a good or poor prognosis. Based on results of the phase III TAILORx ${ }^{3}$ trial, the 21-gene assay is recommended by the NCCN Clinical Practice Guidelines in Oncology (NCCN Guidelines) for Breast Cancer ${ }^{4}$ for predicting the benefit from adding adjuvant chemotherapy to adjuvant endocrine therapy in women with localized hormone receptor (HR)-positive, HER2-negative disease. Generally, poor prognosis is predictive of benefit from chemotherapy (ie, a greater and meaningful absolute risk reduction in cancer recurrence or death) and good prognosis is not.

The TAILORx trial enrolled women aged 18 to 75 years with HR-positive, HER2negative breast cancer measuring 1.1 to $5.0 \mathrm{~cm}$ (or $0.5-1.0 \mathrm{~cm}$ with unfavorable histologic features, defined as intermediate or poor nuclear and/or histologic grade or lymphovascular invasion) without involvement of axillary lymph nodes. ${ }^{5}$ To be eligible, patients had to have undergone complete surgical resection, not have received prior chemotherapy or radiation therapy, and have an anticipated life expectancy of at least 10 years without significant comorbidity. All trial participants had their surgical specimen tested using Oncotype DX. Women with a low RS $(\leq 10)$ were treated with endocrine therapy alone and women with high RS $(>25)$ were treated with adjuvant chemotherapy followed by endocrine therapy. Women with an intermediate RS (11-25) underwent randomization to adjuvant chemotherapy followed by endocrine therapy or endocrine therapy alone. Findings showed that invasive disease-free survival (DFS), freedom from recurrence at a distant site, and overall survival were not different for women with an intermediate RS treated with endocrine therapy alone versus chemoendocrine therapy, and both groups matched outcomes of those with low RS treated with endocrine therapy. ${ }^{3}$ Notably, $78 \%$ with low RS, $74 \%$ with intermediate RS, and $43 \%$ with high RS were found to have low clinical risk as determined by Adjuvant! Online. 
Recently, a secondary analysis of TAILORx evaluated whether the level of clinical risk provided additional information regarding prognosis for recurrence and predicted benefit for chemotherapy. ${ }^{6}$ In this analysis, patients at high clinical risk showed a greater rate of recurrence, second primary cancers, and death, demonstrating the prognostic value of clinical risk. However, among women with RS 11-25, level of clinical risk did not predict benefit of chemotherapy. This analysis is limited by the limitations of the TAILORx trial, which did not prospectively incorporate standard-ofcare clinical risk assessment to guide treatment decisions. Therefore, we believe a more appropriate question and design would ask whether routine addition of molecular prediction improves patient outcomes over use of clinical risk prediction alone.

For example, the MINDACT ${ }^{7}$ trial appropriately tested the MammaPrint molecular assay for clinical utility compared with standard of care clinical prediction. This trial enrolled women aged 18 to 70 years with histologically confirmed stage T1, T2, or operable T3 invasive breast cancer. In the original design, participants with involved lymph nodes were excluded; however, the protocol was later liberalized to allow patients with $\leq 3$ positive axillary lymph nodes to enroll. HR and HER2 status were not specified in the inclusion criteria, but $88.4 \%$ of patients had HR-positive and $90.3 \%$ had HER2-negative disease.

Patients enrolled in the MINDACT trial were separated into 4 main risk groups based on low versus high clinical risk as determined by Adjuvant! Online (version 8.0), and low versus high genomic risk as determined by the MammaPrint assay. ${ }^{7}$ Women with discordant clinical and genomic risk were randomized to receive adjuvant chemotherapy followed by endocrine therapy or endocrine therapy alone, whereas those with concordant low clinical and genomic risk received endocrine therapy alone and those with concordant high clinical and genomic risk were treated with adjuvant chemotherapy followed by endocrine therapy. In this trial, chemotherapy showed no benefit in the primary outcome of survival without distant metastasis for women with either low clinical/high genomic risk or high clinical/low genomic risk. Similarly, no difference was seen in overall survival. However, patients with high clinical/low genomic risk treated with chemotherapy based on clinical risk showed improved 5-year DFS (93.3\% vs $90.3 \%$ using genomic risk; hazard ratio, $0.64 ; P=.03$ ), a result unlikely to translate to an overall survival benefit.

It is important to consider clinical risk in trials that attempt to validate the utility of novel molecular predictive tools over clinical risk alone. Multiple studies have shown the Oncotype DX RS can be estimated using routine pathologic markers. ${ }^{8-10}$ A series of prognostic equations have been developed using pathologic markers, including tumor grade, estrogen receptor and progesterone receptor status, HER2 expression, and Ki-67 immunohistochemistry. Concordance between RS calculated using these equations and Oncotype DX RS was $>95 \% .{ }^{9,10}$ This would suggest that the Oncotype DX assay may be rendered unnecessary. Therefore, using Oncotype DX in lieu of other prognostic variables could increase the cost of care and delay treatment without clear benefit. The recent secondary analysis using TAILORx data to show the predictive utility of the assay did not investigate whether the assay outperforms clinical risk but rather showed that there is minimal utility of clinical risk compared with Oncotype DX. ${ }^{6}$ This result raises the question of whether the converse could also be true (which was not tested in this trial). Finally, some experts have noted subgroup findings in TAILORx that seem to show the value of chemotherapy in young patients with large tumors irrespective of RS. ${ }^{11}$ This again suggests the need to incorporate clinical features.

For this reason, based on currently available data, we believe a more reasonable approach to guide de-escalation would be to use clinical risk alone, although some clinicians have advocated for an approach akin to that used in the MINDACT study. ${ }^{12}$ Specifically, patients identified with high clinical risk could undergo genomic testing to determine whether they would benefit from chemotherapy. If the genomic

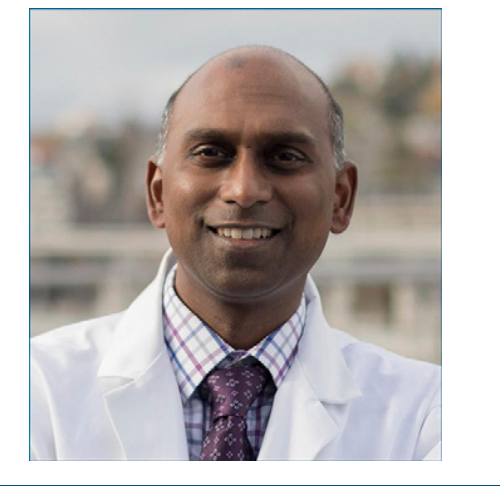

\section{V.K. GADI, MD, PhD}

V.K. Gadi, MD, PhD, is a medical oncologist and Associate Professor of Medicine at the University of Washington. He also holds appointments in the Clinical Research and Public Health Sciences Divisions at the Fred Hutchinson Cancer Research Center. He is a translational breast cancer researcher and clinician.

Dr. Gadi has disclosed that he receives grant/research support from Roche and is a consultant for Seattle Genetics. He is a founder of SEngine Precision Medicine. 


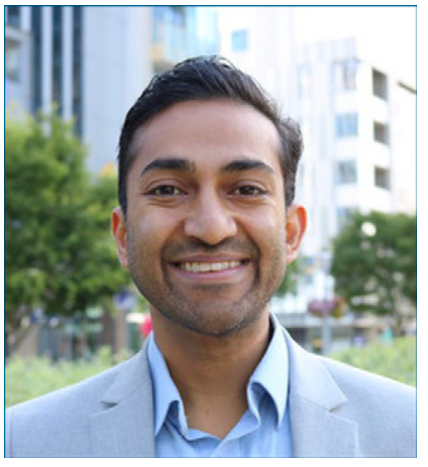

VINAY PRASAD, MD, MPH

Vinay Prasad, MD, MPH, is a hematologistoncologist and Associate Professor of Medicine at Oregon Health and Science University. He is Assistant Program Director of the Hematology Oncology Fellowship Program. He also holds an appointment as a Senior Scholar in the Center for Health Care Ethics. He studies cancer drugs, health policy, and evidence-based medicine. $\mathrm{He}$ is an author of more than 200 academic articles, coauthor of the book Ending Medical Reversal, and author of the forthcoming book Malignant.

Dr. Prasad has disclosed that he receives royalties from his book, Ending Medical Reversal; his work is funded by the Laura and John Arnold Foundation; he receives honoraria for grand rounds/lectures from several universities, medical centers, and professional societies and payments for contributions to Medscape; and he is the maker of the Plenary Session Podcast, which is funded through Patreon. risk is predicted to be low, then despite high clinical risk, the value of chemotherapy is approximately a $3 \%$ absolute increase in DFS but no benefit for distant metastasis-free survival. Some believe this benefit is marginal, which justifies omission of treatment. ${ }^{13}$ However, a similar numerical difference has also been used to justify approval of other breast cancer drugs. For example, this is greater than the $2.3 \%$ absolute difference in 2-year invasive DFS shown by neratinib ${ }^{14}$ and the $0.9 \%$ improvement in invasive DFS shown by pertuzumab in the adjuvant setting, ${ }^{15}$ both of which led to FDA approval. Thus, MINDACT asks clinicians and patients to use a molecular tool to identify a population that may choose to forgo a benefit larger than that offered by 2 approved anticancer medications that other patients may request. This decision should be explained to patients and individualized. Finally, MINDACT confirms a lack of utility for molecular prognostication in patients with low clinical risk because those with discordant clinical and genomic risk did not show benefit from adjuvant chemotherapy for the primary endpoint of freedom from distant metastasis.

The lesson from these studies is that trials must test the clinically relevant question of whether genomic prediction improves decision-making beyond use of the best available clinical algorithms. This is a lesson that should be extended beyond breast cancer. It is possible that a future genomic test for colon cancer may identify which patients benefit from chemotherapy in the adjuvant space; however such a test would have limited applicability if providers were discouraged from using nodal involvement (stage II vs III)—a well-established predictive marker-in guiding therapy. The question facing such a theoretical genomic test is whether it improves classification beyond all clinical predictive markers used in practice. Thus, we hope more trials mimic the design of MINDACT rather than TAILORX.

\section{References}

1. Ravdin PM, Siminoff LA, Davis GJ, et al. Computer program to assist in making decisions about adjuvant therapy for women with early breast cancer. J Clin Oncol 2001;19:980-991.

2. Wishart GC, Azzato EM, Greenberg DC, et al. PREDICT: a new UK prognostic model that predicts survival following surgery for invasive breast cancer. Breast Cancer Res 2010;12:R1.

3. Sparano JA, Gray RJ, Makower DF, et al. Adjuvant chemotherapy guided by a 21-gene expression assay in breast cancer. N Engl J Med 2018;379:111-121.

4. Gradishar WJ, Anderson BO, Abraham J, et al. NCCN Clinical Practice Guidelines in Oncology: Breast Cancer. Version 3.2019. Accessed October 24, 2019. To view the most recent version, visit NCCN.org.

5. Sparano JA, Gray RJ, Makower DF, et al. Prospective validation of a 21-gene expression assay in breast aancer. N Engl J Med 2015;373:2005-2014.

6. Sparano JA, Gray RJ, Ravdin PM, et al. Clinical and genomic risk to guide the use of adjuvant therapy for breast cancer. N Engl J Med 2019;380:2395-2405.

7. Cardoso F, van't Veer LJ, Bogaerts J, et al. 70-Gene signature as an aid to treatment decisions in earlystage breast cancer. N Engl J Med 2016;375:717-729.

8. Allison $\mathrm{KH}$, Kandalaft PL, Sitlani $\mathrm{CM}$, et al. Routine pathologic parameters can predict Oncotype DX recurrence scores in subsets of ER positive patients: who does not always need testing? Breast Cancer Res Treat 2012;131:413-424.

9. Klein ME, Dabbs DJ, Shuai Y, et al. Prediction of the Oncotype DX recurrence score: use of pathologygenerated equations derived by linear regression analysis. Mod Pathol 2013;26:658-664.

10. Bhargava R, Clark BZ, Dabbs DJ. Breast cancers with Magee equation score of less than 18, or 18-25 and mitosis score of 1, do not require Oncotype DX testing: a value study. Am J Clin Pathol 2019;151: 316-323.

11. Poorvu PD, Gelber SI, Rosenberg SM, et al. Selection for Oncotype Dx testing among young women with early-stage ER+/HER2- breast cancer [abstract]. J Clin Oncol 2018;36(Suppl):Abstract 533.

12. Brufsky A. Deciphering genomic testing options for diverse patient populations in early-stage breast cancer. Published November 16, 2018. Available at: https://cancerletter.com/articles/20181116_4/. Accessed November 7, 2019.

13. Blok EJ, van de Velde CJ, Smit VT. 70-Gene signature in early-stage breast cancer. N Engl J Med 2016 375:2199-2201.

14. Martin M, Holmes FA, Ejlertsen B, et al. Neratinib after trastuzumab-based adjuvant therapy in HER2 positive breast cancer (ExteNET): 5-year analysis of a randomised, double-blind, placebo-controlled phase 3 trial. Lancet Oncol 2017;18:1688-1700.

15. von Minckwitz G, Procter M, de Azambuja E, et al. Adjuvant pertuzumab and trastuzumab in early HER2-positive breast cancer. N Engl J Med 2017;377:122-131. 